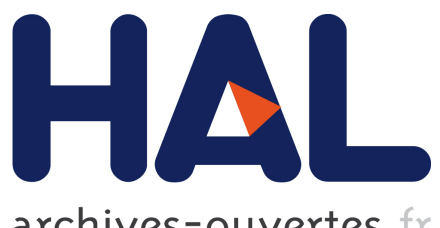

archives-ouvertes

\title{
Vestibular stimulation makes people more egocentric
}

Christophe Lopez, Anastasia Pavlidou, Elisa Raffaella Ferrè, Elisa Ferr

\section{To cite this version:}

Christophe Lopez, Anastasia Pavlidou, Elisa Raffaella Ferrè, Elisa Ferr. Vestibular stimulation makes people more egocentric. Cortex, Elsevier, 2018, 101, pp.302 - 305. 10.1016/j.cortex.2017.12.005 . hal-01794683

\section{HAL Id: hal-01794683 \\ https://hal-amu.archives-ouvertes.fr/hal-01794683}

Submitted on 6 May 2019

HAL is a multi-disciplinary open access archive for the deposit and dissemination of scientific research documents, whether they are published or not. The documents may come from teaching and research institutions in France or abroad, or from public or private research centers.
L'archive ouverte pluridisciplinaire HAL, est destinée au dépôt et à la diffusion de documents scientifiques de niveau recherche, publiés ou non, émanant des établissements d'enseignement et de recherche français ou étrangers, des laboratoires publics ou privés. 


\title{
Vestibular stimulation makes people more egocentric
}

\author{
Anastasia Pavlidou $^{a}$, Elisa Raffaella Ferrè ${ }^{b, 1}$ and Christophe Lopez ${ }^{a, *, 1}$ \\ a Aix Marseille University, CNRS, LNIA, FR3C, Marseille, France \\ ${ }^{\mathrm{b}}$ Department of Psychology, Royal Holloway University of London, Egham, United Kingdom
}

\section{Introduction}

We experience our environment from an egocentric, firstperson perspective. Only during rare conditions, such as Out-of-Body Experiences (OBEs), do people claim they experience their environment from a disembodied perspective (Ionta et al., 2011). Experimental inductions of multisensory conflicts demonstrate that the experience of an egocentric perspective should not be taken for granted, as it is dynamically shaped by visual, somatosensory and vestibular signals (Ehrsson, 2007; Pfeiffer et al., 2013).

The vestibular system encodes self-orientation and selfmotion with respect to gravity. Critically, vestibular disorders may evoke OBEs (Lopez \& Elziere, 2017) and direct stimulation of the temporo-parietal vestibular cortex may induce a disembodied perspective (Blanke, Ortigue, Landis, \& Seeck, 2002). This suggests an important vestibular contribution in anchoring the visuo-spatial perspective to the body.
Humans have a natural tendency to spontaneously adopt the visuo-spatial perspective of others in their environment. In the "dot-counting task" (Samson, Apperly, Braithwaite, Andrews, \& Bodley Scott, 2010), participants determine if the number of dots in a visual scene match a number presented at the start of the trial. The visual scene includes a taskirrelevant avatar. Under conditions where the avatar "sees" a number of dots incongruent with the number of dots visible from the participants' viewpoint, response times increase. This reflects the implicit simulation of the avatar's viewpoint, referred to as "altercentric intrusion". Here we combined the dot-counting task with low-intensity galvanic vestibular stimulation (GVS), known to modulate self-perception in a polarity-dependent manner. Left-anodal/right-cathodal GVS activates predominantly the right hemisphere, whereas rightanodal/left-cathodal GVS activates predominantly the left hemisphere (Fink et al., 2003). Since the vestibular system has been shown to contribute in anchoring the visuo-spatial perspective to the body, we hypothesize that altering its activity during the dot-counting task will disrupt the natural tendency to altercentric intrusions by interfering with multisensory processing in brain regions involved in perspective taking, such as the temporo-parietal cortex.

\section{Methods}

Thirty-two right-handed volunteers were included (Study 1: $n=16,22.3 \pm 4.0$ years; Study $2: n=16,24.2 \pm 4.2$ years). The sample size for each study was a priori decided based on a power analysis (see Supplementary Methods).

\footnotetext{
* Corresponding author. Laboratoire de Neurosciences Intégratives et Adaptatives - UMR 7260, Aix Marseille University, Centre National de la Recherche Scientifique (CNRS), Centre Saint-Charles, Fédération de Recherche 3C - Case B, 3, Place Victor Hugo, 13331, Marseille Cedex 03, France.

E-mail addresses: christophe.lopez@univ-amu.fr, christophe.g.lopez@gmail.com (C. Lopez).

1 These authors share senior authorship. https://doi.org/10.1016/j.cortex.2017.12.005
} 
A

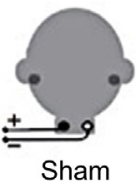

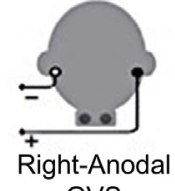
GVS

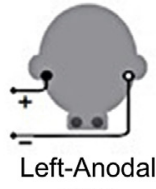

GVS
B
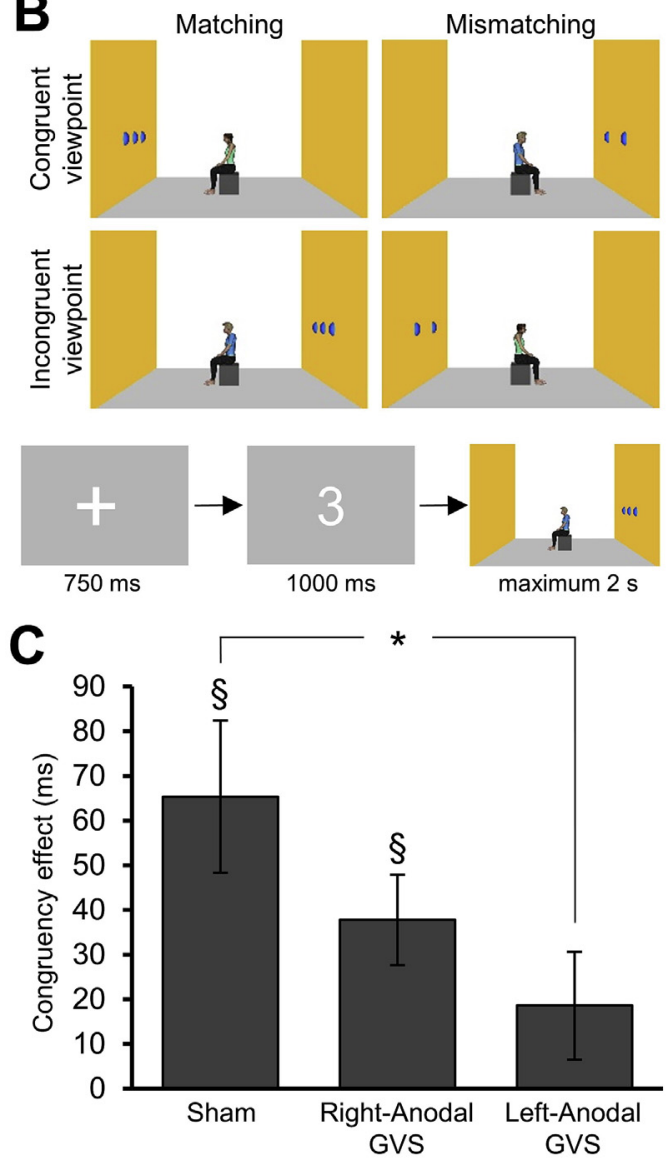

D

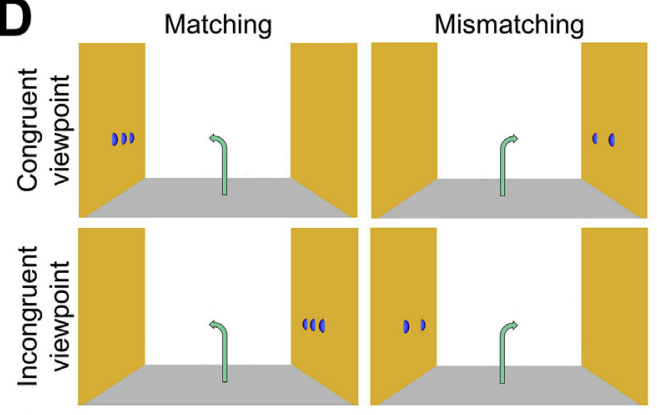

E

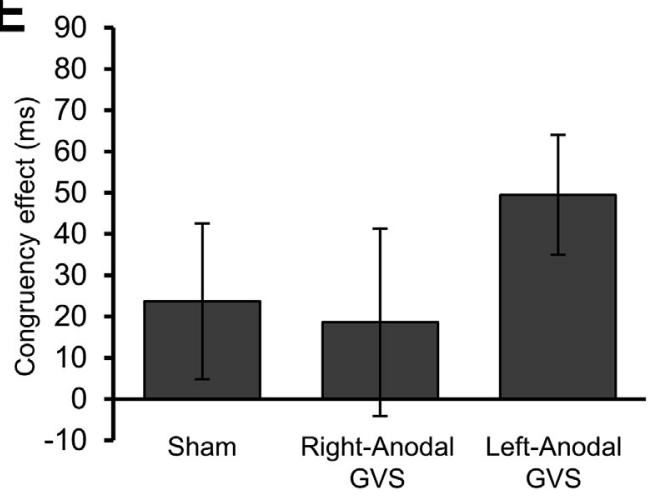

A $1 \mathrm{~mA}$ square wave GVS was delivered through a pair of carbon-rubber electrodes placed over the left and right mastoid processes, using both left-anodal/right-cathodal and right-anodal/left-cathodal configurations (Fig. 1A). Sham stimulation was also delivered at the same intensity through electrodes placed at the base of the neck (Ferre, Lopez, \& Haggard, 2014).

Visual stimuli, presented on a computer screen, consisted of a 3D room whose left and right walls contained from 0 to 3 blue balls. In Study 1, a task-irrelevant avatar in the middle of the room faced either the left wall (8 participants) or right wall (8 participants) (Fig. 1B). The manipulation of the number of balls aligned to the left and right walls created scenarios where the avatar and the participant "saw" the same number (congruent viewpoints) or different number (incongruent viewpoints) of balls. A number was presented on the screen the start of each trial, after which participants indicated whether this number matched (i.e., matching trials) or mismatched the total number of blue balls they saw. Participants completed three blocks of 78 trials during which left-anodal/rightcathodal GVS, right-anodal/left-cathodal GVS or sham stimulation was applied in a quasi-balanced order.

In Study 2, the avatar was replaced by an arrow (Fig. 1D), to exclude GVS effects on visuo-spatial attention and on the ability to inhibit conflicting information (Nielsen, Slade, Levy,

Fig. 1 - Experimental setup and results. (A) Sham stimulation, right-anodal/left-cathodal galvanic vestibular stimulation (GVS), and left-anodal/right-cathodal GVS were delivered in separate blocks through carbon-rubber electrodes placed over the mastoid processes for GVS, or at the base of the neck for sham stimulation. (B) Study 1: Visual stimuli presented either a congruent or an incongruent viewpoint of the avatar's perspective to that of the participants'. Each trial started with a fixation cross (750 $\mathrm{msec})$, followed by a number $(1000 \mathrm{msec})$ and then the visual scene (maximum time 2000 msec). Participants had to indicate with a button press whether the number of blue balls observed from their viewpoint matched or mismatched the number presented at the start of the trial. The example illustrates a matching trial with an incongruent viewpoint. (C) Bar plot represents the average congruency effect in milliseconds (difference between the response times in the trials with incongruent and congruent viewpoint) calculated for the matching trials during sham stimulation, right-anodal/left cathodal GVS and left-anodal/right cathodal GVS for Study 1. ${ }^{*}$ denotes a significant difference between stimulation conditions. $\S$ denotes significant differences with respect to zero. Vertical bars represent the standard error of mean. (D) Study 2: Visual stimuli presented either a congruent or an incongruent viewpoint of the arrow's perspective to that of the participants'. The timing of each trial and procedure was the same as in Study 1. (E) Bar plot represents the average congruency effect in milliseconds calculated for the matching trials during sham stimulation, rightanodal/left cathodal GVS and left-anodal/right cathodal GVS for Study 2. Vertical bars represent the standard error of mean. 
\& Holmes, 2015; Santiesteban, Catmur, Hopkins, Bird, \& Heyes, 2014). Experimental design and procedures were otherwise identical to Study 1.

\section{Results}

To quantify the extent to which the avatar's viewpoint interfered with the participants' viewpoint, we calculated a Congruency Effect (CE) as the difference in response times between incongruent and congruent viewpoints for the matching trials (Nielsen et al., 2015). Mismatching trials were discarded from the main analysis following previous procedures (Samson, Apperly, Braithwaite, Andrews, \& Bodley Scott, 2010; see Supplementary Methods). A repeated-measures ANOVA with Stimulation (left-anodal/right-cathodal GVS, right-anodal/left-cathodal GVS and sham stimulation) as a within-subject factor and Avatar orientation (facing left, facing right) as a between-subject factor revealed that the CE was significantly modulated by Stimulation $\left(F_{2,28}=3.50, p<.05\right.$, $\eta_{P}^{2}=.20$; Fig. 1C). Post-hoc analysis corrected for multiple comparisons showed a significantly reduced CE for left anodal/right cathodal GVS compared to sham stimulation (Bonferroni test: $p=.041$ ). By contrast, the CE for right anodal/ left cathodal GVS did not differ from sham stimulation (Bonferroni test: $p=.395$ ) and left anodal/right cathodal GVS (Bonferroni test: $p=.868$ ). There was no effect of Avatar orientation and no Stimulation $\times$ Avatar orientation interaction (all $F<3.0$ and $p>.1$ ). Critically, the CE was significantly higher than zero for right-anodal/left-cathodal GVS $\left(\mathrm{t}_{15}=3.53\right.$, $p<.005)$ and sham stimulation $\left(t_{15}=3.78, p<.005\right)$, indicating, as expected, altercentric intrusion. By contrast, for leftanodal/right-cathodal GVS the CE did not differ from zero $\left(t_{15}=1.43, p=.17\right)$, suggesting a suppression of altercentric intrusion.

A similar analysis applied to the CE for the number of errors revealed no effect of Stimulation, Avatar orientation and interaction (all $F<2.2$ and $p>.1$; Supplementary Fig. 1).

Although mismatching trials are commonly discarded from analysis (Supplementary Methods), we have analysed them to investigate any potential GVS influence on visuospatial attention. This analysis revealed no effect of Stimulation on the CE for mismatching trials $\left(F_{2,28}=.64, p=.533\right.$, $\left.\eta^{2}=.04\right)$. Similarly, no effect of Stimulation on the CE emerged on Study $2\left(F_{2,28}=1.85, p=.174, \eta_{P}^{2}=.11\right.$; Fig. $\left.1 \mathrm{E}\right)$. This pattern of results confirms that the reduction in CE for the matching trials was not due to any GVS effect on visuospatial attention, or on the ability to inhibit conflicting information (see Supplementary Results).

\section{Discussion and conclusion}

Left anodal/right cathodal GVS decreased altercentric intrusion compared to sham stimulation. In right-handed participants, such GVS polarity is known to activate the temporoparietal and parieto-insular cortex with a right hemisphere dominance (Fink et al., 2003; Lopez, Blanke, \& Mast, 2012) and there is an overall right-sided dominance of vestibular information processing (Dieterich, Kirsch, \& Brandt, 2017).
Importantly, both areas are crucial for embodiment and perspective taking: lesions and epilepsy affecting these brain regions evoke the sensation of perceiving the environment from a disembodied perspective (Blanke et al., 2002; Ionta et al., 2011). Accordingly, theta oscillations originating from the right temporo-parietal junction reflect an imaginary disembodied perspective (Wang, Callaghan, Gooding-Williams, McAllister, \& Kessler, 2016). Thus, GVS might have modulated activity in the right temporo-parietal junction, promoting an egocentric perspective.

Disruption to the multisensory processes involved in embodied perspective - as in OBEs - might require stronger current intensities than the one used in this study. GVS below $1 \mathrm{~mA}$ improves cognitive and sensorimotor performances (Wilkinson et al., 2014) and promotes interpretation of tactile stimuli from an egocentric perspective (Ferre et al., 2014). Thus, low-intensity GVS used in our study may have increased the natural role of the vestibular system in anchoring the visuo-spatial perspective to the body.

Importantly, the decreased altercentric intrusion by GVS is not due to redirection of visuo-spatial attention by GVS or avatar orientation. Results from Study 2 indicate that the influence of GVS on the CE is specific to the avatar stimuli, to which mental states can be ascribed. Avatars and arrows redirect spatial attention to one side. For example, Santiesteban et al. (2014) found altercentric interference for arrow stimuli, concluding that the $C E$ is due to general attentional processes. By contrast, Nielsen et al. (2015) found a weaker response to the incongruence of the viewpoint of an arrow versus an avatar. Recent evidence show that altercentric intrusions do not arise because avatars prompt a shift in spatial attention, but rather because participants attribute mental states to avatars (Furlanetto, Becchio, Samson, \& Apperly, 2016).

Our results highlight the crucial, yet so far neglected, contribution of vestibular inputs in maintaining an embodied first-person perspective. The right temporo-parietal junction is potentially involved in this process, integrating signals from different sensory pathways. Disruption in the integration of these signals leads to a disembodied self.

\section{Acknowledgements}

The research leading to these results has received funding from the People Programme (Marie Curie Actions) of the European Union's Seventh Framework Programme (FP7/ 2007-2013) under REA grant agreement number 333607 ("BODILYSELF: vestibular and multisensory investigations of bodily self-consciousness"). C.L. and A.P. are supported by the Volkswagen Foundation (grant number 89434: "Finding Perspective: Determining the embodiment of perspectival experience").

\section{Supplementary data}

Supplementary data related to this article can be found at https://doi.org/10.1016/j.cortex.2017.12.005. 


\section{R E F E R E N C E S}

Blanke, O., Ortigue, S., Landis, T., \& Seeck, M. (2002). Stimulating illusory own-body perceptions. Nature, 419(6904), 269-270. https://doi.org/10.1038/419269a.

Dieterich, M., Kirsch, V., \& Brandt, T. (2017). Right-sided dominance of the bilateral vestibular system in the upper brainstem and thalamus. Journal of Neurology. https://doi.org/ 10.1007/s00415-017-8453-8.

Ehrsson, H. H. (2007). The experimental induction of out-of-body experiences. Science, 317(5841), 1048. https://doi.org/10.1126/ science.1142175.

Ferre, E. R., Lopez, C., \& Haggard, P. (2014). Anchoring the self to the body: Vestibular contribution to the sense of self. Psychological Science, 25(11), 2106-2108. https://doi.org/10.1177/ 0956797614547917.

Fink, G. R., Marshall, J. C., Weiss, P. H., Stephan, T., Grefkes, C., Shah, N. J., et al. (2003). Performing allocentric visuospatial judgments with induced distortion of the egocentric reference frame: An fMRI study with clinical implications. NeuroImage, 20(3), 1505-1517.

Furlanetto, T., Becchio, C., Samson, D., \& Apperly, I. (2016). Altercentric interference in level 1 visual perspective taking reflects the ascription of mental states, not submentalizing. Journal of Experimental Psychology: Human Perception and Performance, 42(2), 158-163. https://doi.org/10.1037/ xhp0000138.

Ionta, S., Heydrich, L., Lenggenhager, B., Mouthon, M., Fornari, E., Chapuis, D., et al. (2011). Multisensory mechanisms in temporo-parietal cortex support self-location and first-person perspective. Neuron, 70(2), 363-374. https://doi.org/10.1016/ j.neuron.2011.03.009.

Lopez, C., Blanke, O., \& Mast, F. W. (2012). The human vestibular cortex revealed by coordinate-based activation likelihood estimation meta-analysis. Neuroscience, 212, 159-179. https:// doi.org/10.1016/j.neuroscience.2012.03.028.
Lopez, C., \& Elziere, M. (2017). Out-of-body experience in vestibular disorders - a prospective study of 210 patients with dizziness. Cortex; A Journal Devoted to the Study of the Nervous System and Behavior. https://doi.org/10.1016/ j.cortex.2017.05.026.

Nielsen, M., Slade, L., Levy, J. P., \& Holmes, A. (2015). Inclined to see it your way: Do altercentric intrusion effects in visual perspective taking reflect an intrinsically social process? Quarterly Journal of Experimental Psychology, 68(10), 1931-1951. https://doi.org/10.1080/17470218.2015.1023206.

Pfeiffer, C., Lopez, C., Schmutz, V., Duenas, J. A., Martuzzi, R., \& Blanke, O. (2013). Multisensory origin of the subjective firstperson perspective: Visual, tactile, and vestibular mechanisms. PLoS One, 8(4), e61751. https://doi.org/10.1371/ journal.pone.0061751.

Samson, D., Apperly, I. A., Braithwaite, J. J., Andrews, B. J., \& Bodley Scott, S. E. (2010). Seeing it their way: Evidence for rapid and involuntary computation of what other people see. Journal of Experimental Psychology: Human Perception and Performance, 36(5), 1255-1266. https://doi.org/10.1037/ a0018729.

Santiesteban, I., Catmur, C., Hopkins, S. C., Bird, G., \& Heyes, C. (2014). Avatars and arrows: Implicit mentalizing or domaingeneral processing? Journal of Experimental Psychology: Human Perception and Performance, 40(3), 929-937. https://doi.org/ 10.1037/a0035175.

Wang, H., Callaghan, E., Gooding-Williams, G., McAllister, C., \& Kessler, K. (2016). Rhythm makes the world go round: An MEGTMS study on the role of right TPJ theta oscillations in embodied perspective taking. Cortex; A Journal Devoted to the Study of the Nervous System and Behavior, 75, 68-81. https:// doi.org/10.1016/j.cortex.2015.11.011.

Wilkinson, D., Zubko, O., Sakel, M., Coulton, S., Higgins, T., \& Pullicino, P. (2014). Galvanic vestibular stimulation in hemispatial neglect. Frontiers in Integrative Neuroscience, 8, 4. https:// doi.org/10.3389/fnint.2014.00004. 